\title{
EQUATIONAL AXIOMS FOR CLASSES OF LATTICES
}

\author{
BY KIRBY A. BAKER ${ }^{1}$
}

Communicated by R. S. Pierce, July 27, 1970

1. Equational axiom problems. This note presents a general method for solving a number of problems in the equational theory of lattices. Current interest in this theory stems from Jónsson's important discovery [7], building on earlier work of Birkhoff, that a lattice sharing the algebraic identities common to a given class $K$ of lattices is more tightly bound to $K$ than would be expected for most other kinds of algebraic systems. In this context, a natural question for any such class $K$ is the following "axiom problem."

A.P. $(K)$ : Find a set of equational axioms for $K$, i.e., $a$ set $\Sigma$ of identities, common to the members of $K$, of which all other such identities are lattice-theoretic consequences.

An equivalent requirement on $\Sigma$ is that the class of lattices defined by $\Sigma$ coincide with $K^{e}$, the smallest equational class (class definable by identities) containing $K$.

The problem A.P.(K) is to be viewed as a practical one; the solution is to be constructed explicitly starting from some given definition of $K$. McKenzie [9], for example, has given just such an explicit solution of A.P. $(\{L\})$ for each finite lattice $L$.

The general method to be developed below solves the axiom problems of all classes of lattices in the following list, among many others.

(a) $P P$, the class of all projective planes (viewed as lattices of flats). More generally,

(b) $P P(\varepsilon)$, the class of projective planes subject to a given list $\varepsilon$ of excluded configurations. An example is the class of Desarguesian planes, for which solutions to the axiom problem have been given by Schützenberger [10] and Jónsson [6, Theorem 7.1].

(c) $L t h(m)$, the class of all lattices of length at most $m$, i.e., lattices in which the longest chain has at most $m+1$ elements. This axiom problem was posed by Jónsson, who later solved the case $m=2$ [8].

(d) Wth $(m)$, the class of all lattices of width at most $m$, i.e., lattices

AMS 1970 subject classifications. Primary 06A20, 08A15; Secondary 06A30, 08A20.

Key words and phrases. Equational class of lattices, variety of algebras, algebraic identity, lattice polynomial, universal disjunction of equations.

1 Research supported in part by N.S.F. grants \#GP-13164 and \#GP-23113.

Copyright @ 1971, American Mathematical Society 
in which every totally unordered subset has at most $m$ elements. This axiom problem was also posed by Jónsson; the case $m=2$ was solved by McKenzie [10].

(e) $\operatorname{Br}(m)$, the class of all lattices of breadth at most $m$, i.e., lattices such that every join of $m+1$ elements is redundant.

(f) $P l$, the class of "planar" lattices, i.e., lattices which can be order-embedded in a product of two chains.

(g) $K_{1} \cup K_{2}$, the union of two equational classes of lattices, each defined by a given finite set of identities (McKenzie).

Additionally, Wille [12] raises the topic of equational axioms for the classes $\boldsymbol{M} \cap L t h(m)$ and $\boldsymbol{M} \cap W t h(m)$ of modular lattices of length or width at most $m$. (Here $M$ denotes the class of all modular lattices.) Jónsson [8] in effect solves the case A.P.( $M \cap W t h(3))$. The promised solution of A.P. $(K)$ for any of the nonmodular classes $K$ of $(\mathrm{c})-(\mathrm{g})$ above will yield a solution to A.P. $(M \cap K)$ when augmented by the modular identity.

For each of the axiom problems previously solved, the axioms given have been finite in number. McKenzie [9] and the author [1] have, however, given examples of classes $K$ for which A.P.(K) has no finite solution. In fact, it can be shown that, in at least these specific cases, no finite solution exists: $L t h(m)$ for $m \geqq 4 ; W t h(m)$ for $m \geqq 4$; $M \cap W t h(m)$ for $m \geqq 5 ; \operatorname{Br}(m)$ and $\operatorname{M\cap } \cap \operatorname{Br}(m)$ for $m \geqq 2 ; P l$; and $K_{1} \cup K_{2}$ for certain equational classes $K_{1}, K_{2}$ each defined by a single identity. On the other hand, $P P$ and $M \cap L t h(m)$, for all $m$, do give finite solutions. (Details will appear elsewhere.) The general method of $\S \S 2-3$ below for solving axiom problems always yields an infinite list of identities, reducible (not necessarily constructively) to a finite list when a finite solution exists.

The methods of this paper can be generalized to solve axiom problems relative to any equational class of algebras to which Jónsson's results of [7] apply. Especially promising are applications to orthomodular lattices, Heyting algebras, and lattice-ordered groups. [5].

A general reference for lattice theory is [3]; for universal algebra,

2. A general problem. A common method of solution to all the axiom problems listed in $\S 1$ is to be found at the end of this twisting trail of reasoning:

(1) The most powerful tool relevant to such problems is Jónsson's main theorem of [7].

(2) The classes of lattices to which Jónsson's theorem applies most naturally are those which are closed under the formation of sub- 
lattices, homomorphic images, and ultraproducts. Following the ad hoc terminology of [1], let us call such classes "stable."

(3) It is a result in model theory that a stable class is definable by sentences of a simple form, namely by universally quantified disjunctions of polynomial equations (UDE's).

(4) Happily, most of the classes of lattices on our list are stable, and it is possible to give explicit defining UDE's. The other classes can be expanded to stable ones without changing their axiom problems.

(5) Therefore, all the listed axiom problems are but instances of the axiom problem for a much more general class, namely, the problem A.P.(Lat $\Delta$ ), where $\Delta$ is any given set of UDE's and Lat $\Delta$ denotes the class of all lattices satisfying all the sentences in $\Delta$ (lattice models of $\Delta)$.

(6) This very general axiom problem can immediately be reduced to the case where $\Delta$ consists of a single UDE $\delta$. A solution to this reduced problem will be constructed in the next section.

Amplification for (1), (3), (4), (6). For (1). A statement of Jónsson's result of $[7, \S 3]$, adapted for our purposes, is as follows. (For terminology, see [3] or [5].)

ThEOREM 2.1 (Jónsson). Let $\mathrm{S}$ be the class of all subdirectly irreducible lattices. If $K$ is stable, then $S \cap K^{e}=S \cap K$; in fact, $K^{\circ}$ is the unique equational class $E$ such that $S \cap E=S \cap K$.

For (3). A class closed under formation of ultraproducts, sublattices, and isomorphic images is axiomatic [5, Corollary 1, p. 258]; an axiomatic class closed under formation of sublattices and homomorphic images is definable by positive universal sentences [5, Corollary 2, p. 275], each of which is trivially equivalent to a conjunction of UDE's. Conversely, if $\Delta$ is a set of UDE's, Lat $\Delta$ is stable. Any identity, of course, is trivially a UDE.

For (4). The key method is to apply the following principle from [1]. If $P$ is any finite partly ordered set and $N(P)$ is the class of all lattices which contain no order-isomorphic copy of $P$, then $N(P)$ is stable. For example, $L t h(m)=N\left(C_{m+2}\right)$, where $C_{m+2}$ is an $(m+2)$-element chain; Wth $(m)=N\left(U_{m+1}\right)$, where $U_{m+1}$ is a totally unordered set of $m+1$ elements; $\operatorname{Br}(m)=N\left(B_{m+1}\right)$, where $B_{k}$ is the partly ordered set consisting of 1 -element and $(k-1)$-element subsets of a $k$-element set; $P l=\bigcap_{i} N\left(Q_{i}\right)$, where $Q_{1}, Q_{2}, \cdots$ correspond to "comparability cycles" [2]. For any given $P, N(P)$ can be defined by an explicit UDE as follows.

LEMma 2.2. Let $p(1), \cdots, p(n)$ be the elements of the finite partly or- 
dered set $P$. For each $i$, let $g_{i}(x)$ denote the lattice polynomial $g_{i}\left(x_{1}, \cdots, x_{n}\right)$ $=V_{j} x_{j}$ with $j$ ranging over $\{j: p(j) \leqq p(i)\}$. Then $N(P)=$ Lat $\{\delta\}$, where $\delta$ is the UDE $\left(\forall x_{1}, \cdots, x_{n}\right) O R_{i, k}\left[g_{i}(x)=g_{i}(x) \vee g_{k}(x)\right]$ with $(i, k)$ ranging over $\{(i, k): p(k) \$ P(i)\}$.

Here $O R$ denotes logical disjunction, to avoid confusion with lattice operations. The proof of the lemma is easy and will be omitted.

For a treatment of the nonstable classes $P P$ and $P P(\mathcal{E})$ in terms of $N\left(\right.$ ), see [1]. The only remaining class on our list is $K_{1} \cup K_{2}$; this class is definable by conjunctions of pairs of identities, each of which can be turned into an equivalent UDE by using disjoint sets of variables and taking quantifiers outside.

For (6). If $\Delta$ is a set of UDE's, a solution to A.P.(Lat $\Delta$ ) is obtained merely by lumping together all the identities involved in the solutions $\Sigma_{\delta}$ of the various problems A.P.(Lat $\delta$ ) for $\delta \in \Delta$. This follows from the computation

$\operatorname{Lat}\left(\bigcup_{\delta \in \Delta} \Sigma_{\delta}\right)=\bigcap_{\delta \in \Delta}$ Lat $\Sigma_{\delta}=\bigcap_{\delta \in \Delta}(\text { Lat } \delta)^{e}=\left(\bigcap_{\delta \in \Delta} \text { Lat } \delta\right)^{\theta}=(\text { Lat } \Delta)^{e}$,

in which the third equality is an application of Theorem 2.1, and Lat $\delta$ means Lat $\{\delta\}$.

3. A solution to the general problem. As shown in the last section, it suffices to solve A.P.(Lat $\delta$ ), where $\delta$ is a single UDE. Such a $\delta$ can be written as

$$
\delta=\left(\forall x_{1}, \cdots, x_{m}\right)\left[f_{1}(x)=g_{1}(x) \text { OR } \cdots O R f_{n}(x)=g_{n}(x)\right] .
$$

Replacing $f_{i}, g_{i}$ by $f_{i} \wedge g_{i}, f_{i} \vee g_{i}$ if necessary, we may assume that $f_{i}(\mathrm{x}) \leqq g_{i}(\mathrm{x})$ for all $i$.

The polynomials appearing in the solution of A.P.(Lat $\delta$ ) will be constructed as a three-layered composition, with the inner layer consisting of the $f_{i}$ and $g_{i}$. The middle layer will be built from the polynomials $p_{k}$ defined for $k=0,1,2, \cdots$ by

$p_{k}\left(t ; z_{1}, \cdots, z_{k}\right)=\left(\cdots\left(\left(\left(t \vee z_{1}\right) \wedge z_{2}\right) \vee z_{3}\right) \cdots\right.$, ending in $\cdots) \wedge z_{k}$ for $k$ even, $\left.\cdots\right) \vee z_{k}$ for $k$ odd.

The outer layer will consist simply of $p_{2 n}(t ; z)$ for $n$ as in $\delta$. It will be apparent from the proof of Theorem 3.1 that this type of construction is indeed a natural one.

For $\delta$ as given, then, let us construct for each $k=0,1,2, \cdots$ an identity $\sigma_{k}$, as follows. Let $\boldsymbol{Z}$ be an $n \times k$ array of variables $z_{j}^{i}$, let $r_{i}$ stand for the expression $p_{k}\left(f_{i}(x) ; z_{1}^{i}, \cdots, z_{k}^{i}\right)$, and let $s_{i}$ stand for $p_{k}\left(g_{i}(x) ; z_{1}^{i}, \cdots, z_{k}^{i}\right)$. Let $\sigma_{k}$ be defined by 
$\sigma_{k}=(\forall u, v, x, Z)\left[p_{2 n}\left(u ; r_{1}, s_{1}, \cdots, r_{n}, s_{n}\right)=p_{2 n}\left(v ; r_{1}, s_{1}, \cdots, r_{n}, s_{n}\right)\right]$.

Let $\Sigma_{\delta}$ be the collection $\left\{\sigma_{k}: k=0,1,2, \cdots\right\}$.

THEOREM 3.1. $\Sigma_{\delta}$ solves the axiom problem for the class Lat $\delta$.

The proof, given below, will depend on three properties of the polynomials $p_{k}$. For a lattice $L$ let Quo $(L)$ denote the set of nontrivial "formal quotients" of $L$, i.e., pairs $(a, b) \in L \times L$ with $b>a$, written instead as $b / a$. For $b / a, d / c \in Q u o(L)$, write $b / a \rightarrow d / c$ if there exist $k \geqq 0$ and elements $w_{1}, \cdots, w_{k} \in L$ such that $c=p_{k}\left(a ; w_{1}, \cdots, w_{k}\right)$, $d=p_{k}\left(b ; w_{1}, \cdots, w_{k}\right)$. This is shown without difficulty to be equivalent to the assertion that $d / c$ is weakly projective into $b / a[4$, Definition 2.1].

LEMMA 3.2. Let $L$ be a lattice.

(i) If $c, a_{1}, \cdots, a_{k} \in L$ and $a_{i}=a_{i+1}$ for some $i$, then the value $p_{k}\left(c ; a_{1}, \cdots, a_{k}\right)$ does not depend on the choice of $c$.

(ii) If $d / c \in Q u o(L)$ and $u=c$ or $u=d$, then $p_{k}(u ; c, d, c, d, \cdots, c, d)$ $=u$.

(iii) $\rightarrow$ is a quasi-ordering on $\mathrm{Quo}(L)$ and is directed if $L$ is subdirectly irreducible.

Proof. (i) and (ii) are immediate, as are reflexivity and transitivity in (iii). Directedness is verified as follows. For $b / a \in Q u o(L)$, let $\theta(a, b)$ denote the congruence relation generated by identifying $a$ and $b$. [4, Lemma 2.1] states that if $\theta(c, d) \subseteq \theta(a, b)$, then there exists a chain $c=c_{0}<c_{1}<\cdots<c_{n}=d$ with $b / a \rightarrow c_{i} / c_{i-1}$ for all $i>0$. Further, if $L$ is subdirectly irreducible, there exists a smallest congruence relation $\theta(c, d)$ [3, Corollary 2, p. 140]. Thus, if $b / a, b^{\prime} / a^{\prime}$ are any two given elements of Quo $(L)$, there are corresponding chains $\left\{c_{i}\right\},\left\{c_{j}^{\prime}\right\}$ from $c$ to $d$. Choose $(i, j)$ to be a minimal pair of indices such that $c_{i} \wedge c_{j}^{\prime}>c$. Then for $e=c_{i} \wedge c_{j}^{\prime}, b / a \rightarrow c_{i} / c_{i-1} \rightarrow e / c, b^{\prime} / a^{\prime}$ $\rightarrow c_{j}^{\prime} / c_{j-1}^{\prime} \rightarrow e / c$, so that $e / c$ is an upper bound for $b / a, b^{\prime} / a^{\prime}$ in $Q u o(L)$.

Proof OF TheORem 3.1. We must show that (Lat $\delta)^{e}=$ Lat $\Sigma_{\delta}$. By Theorem 2.1 , it is sufficient to verify that for a subdirectly irreducible lattice $L, L \in$ Lat $\delta$ if and only if $L \in$ Lat $\Sigma_{\delta}$.

Suppose $L \in$ Lat $\delta$. Then for any $k$ and for any substitution of elements for the variables $\left\{x_{i}\right\},\left\{z_{j}^{i}\right\}$, we have the following situation: For some $i$, the evaluated $f_{i}$ and $g_{i}$ are equal. Then the evaluated $r_{i}$ and $s_{i}$ are also equal, and hence by Lemma 3.2-(i), the value of $p_{2 n}\left(c ; r_{1}, s_{1}, \cdots, r_{n}, s_{n}\right)$ does not depend on the choice of $c \in L-$ exactly what $\sigma_{k}$ asserts! Thus $L \in$ Lat $\Sigma_{\delta}$.

Suppose conversely that $L \in$ Lat $\delta$. Then, interpreting variables as 
elements of $L$, we know that there exist $x_{1}, \cdots, x_{m} \in L$ such that $f_{i}(\mathrm{x})<g_{i}(\mathrm{x})$ for all $i$. Write $a_{i}=f_{i}(\mathrm{x}), b_{i}=g_{i}(\mathrm{x})$. By Lemma 3.2-(iii), there exists $d / c \in Q u o(L)$ such that $b_{i} / a_{i} \rightarrow d / c$ for all $i$. Then, for a large enough $k$, there exist suitable $z_{j}^{i} \in L$ such that for all $i, r_{i}$ $=p_{k}\left(a_{i} ; z_{1}^{i}, \cdots, z_{k}^{i}\right)=c, s_{i}=p_{k}\left(b_{i} ; z_{1}^{i}, \cdots, z_{k}^{i}\right)=d$. Setting $u=c, v$ $=d$ and applying Lemma 3.2-(ii), we obtain $p_{2 n}\left(u ; r_{1}, s_{1}, \cdots, r_{n}, s_{n}\right)$ $=c, p_{2 n}\left(v ; r_{1}, s_{1}, \ldots, r_{n}, s_{n}\right)=d$, a violation of $\sigma_{k}$. Therefore $L \in$ Lat $\Sigma_{\delta}$. The proof is thus complete.

\section{REFERENCES}

1. K. A. Baker, Equational classes of modular lattices, Pacific J. Math. 28 (1969), 9-15. MR 39 \#5435.

2. K. Baker, P. Fishburn and F. Roberts, Partial orders of dimension 2, interval orders, and interval graphs, Rand Corp., p. 4376.

3. G. Birkhoff, Lattice theory, 3rd. ed., Amer. Math. Soc. Colloq. Publ., vol. 25, Amer. Math. Soc., Providence, R. I., 1967. MR 37 \#2638.

4. R. P. Dilworth, The structure of relatively complemented lattices, Ann. of Math. (2) 51 (1950), 348-359. MR 11, 489.

5. G. Grätzer, Universal algebra, Van Nostrand, Princeton, N. J., 1968. MR 40 $\# 1320$.

6. B. Jonsson, Modular lattices and Desargues' theorem, Math. Scand. 2 (1954), 295-314. MR 16, 787.

7. - Algebras whose congruence lattices are distributive, Math. Scand. 21 (1967), 110-121. MR 38 \#5689. \#66.

8. - Equational classes of lattices, Math. Scand. 22 (1968), 187-196. MR 40

9. R. McKenzie, Equational bases for lattice theories, Math. Scand. (to appear).

10. - Equational bases and non-modular lattice varieties (to appear).

11. M.-P. Schützenberger, Sur certains axiomes de la theorie des structures, C. R. Acad. Sci. Paris 221 (1945), 218-220. MR 7, 235.

12. R. Wille, Primitive Länge und primitive Weite bei modularen Verbänden, Math. Z. 108 (1969), 129-136. MR 39 \#2672.

University of California, los Angeles, California 90024 